\title{
On the Thermodynamical Equilibrium of Fluids in Gravitational Fields
}

\author{
O. KLEIN \\ Stockholms Högskola, Stockholm, Sweden
}

\begin{abstract}
It is shown that the thermodynamical equilibrium condition for a fluid at rest in a static gravitational field is, apart from the Tolman condition $T\left(g_{44}\right)^{\frac{1}{2}}=$ const., governed by the condition $\alpha\left(g_{44}\right)^{\frac{1}{2}}=$ const., where $\alpha$ is the chemical potential of the fluid (including mass energy) regarded as a function of the space coordinates. This relation constitutes the relativistic generalization of the well-known Gibbs condition for the equilibrium in a gravitational field.
\end{abstract}

$T^{1}$ HE chemical potential introduced into thermodynamics by Gibbs is not only most suited for the expression of chemical equilibria but provides also with the simplest means of expressing the equilibrium condition for a fluid in an external, say gravitational field. In fact, as shown by Gibbs, this condition may be stated in the following way

$$
\mu+M \varphi_{g}=\text { const., }
$$

where $\mu$ is the chemical potential, $M$ the molecular mass and $\varphi_{g}$ the gravitational potential at the place in question. $^{1}$

As an example of the use of this relation we may consider an ideal, monoatomic gas. Here the concentration $C$, the number of particles per unit volume, is related to the chemical potential by means of the following formula

$$
C=\left(2 \pi M \theta / h^{2}\right)^{\frac{3}{2}} e^{\mu / \theta},
$$

where $\theta=k T$ is the temperature modulus and $h$ the Planck constant. By means of (1) we obtain now for the distribution of the gas in a gravitational field

$$
C=C_{0} e^{-M \varphi_{0} / \theta},
$$

$C_{0}$ being the concentration at a place, where $\varphi_{g}$ is taken to be 0 , which is just the ordinary barometer formula.

We shall now find the corresponding statement in Einstein's theory of gravitation. Let us regard the line element given by

$$
d s^{2}=g_{\mu \nu} d x^{\mu} d x^{\nu}
$$

belonging to a static gravitational field, where the $g_{\mu \nu}$ are independent of the suitably chosen time coordinate $x^{4}$. We shall use the following expression for the energy momentum tensor of the fluid ${ }^{2}$

$$
T^{\mu \nu}=(u+p) v^{\mu \nu} v^{\nu}-g^{\mu \nu} p,
$$

where $v^{\mu}$ is the velocity four vector at an arbitrary point of the substance, satisfying the relation

$$
g_{\mu \nu} v^{\mu \nu}=1
$$

${ }^{1} \mathrm{~W}$. Gibbs, Collected Works (Yale University Press, New Haven, 1948) I, p. 144.

${ }^{2}$ R. C. Tolman, Relativity, Thermodynamics and Cosmology (Oxford University Press, London, 1934) p. 269. $u$ the energy density including the mass energy and $p$ the pressure at the same point. We shall now assume that the substance is at rest in the coordinate system chosen, so that

and

$$
v^{1}=v^{2}=v^{3}=0
$$

$$
v^{4}=\frac{1}{\left(g_{44}\right)^{\frac{1}{2}}}=\frac{1}{c}-e^{-v / 2}
$$

where we have put

$$
g_{44}=c^{2} e^{\nu} .
$$

A straightforward calculation then gives

$\operatorname{Div}_{i} T=\frac{1}{(g)^{\frac{1}{2}}} \frac{\partial(g)^{\frac{1}{2}} T_{i}{ }^{\mu}}{\partial x^{\mu}}=-\left(\frac{\partial p}{\partial x_{i}}+\frac{u+p}{2} \frac{\partial \nu}{\partial x_{i}}\right), \quad i=1,2,3$, and since this divergence vanishes on account of the field equations we have as equilibrium condition

$$
\nabla p+(u+p / 2) \nabla \nu=0
$$

where we have used the symbol $\nabla$ to denote the gradient operator corresponding to the space coordinates. ${ }^{3}$ This condition is of course the relativistic analogon to the classical equation

$$
\nabla p+\rho \nabla \varphi_{g}=0,
$$

where $\rho$ is the mass density, which governs the equilibrium in static gravitational fields. We see that with

$$
\nu=\left(2 \varphi_{g} / c^{2}\right) \text { and } u+p=\rho c^{2},
$$

which relations are valid in a weak gravitational field, Eqs. (9) and (10) are, in fact, identical.

Let now $U$ be the inner energy including the mass energy of a volume $V$ of the fluid containing $N$ particles. Then from well-known thermodynamical principles we have

$$
d U=T d S-p d V+\alpha d N
$$

where $S$ is the entropy, and where $\alpha$ is the chemical potential per unit particle including the mass energy. This means that

$$
\alpha=\mu+M c^{2},
$$

${ }^{3}$ See reference 2 , p. 317 , where the same relation is derived on slightly less general assumptions. 
if $\mu$ is the chemical potential as used above in formula (2). The differentials $d U, d S, d V$ and $d N$ denote arbitrary, simultaneous changes in the respective quantities. Now for fluids, whose surface energy may be neglected $U / N$ and $S / N$ are both functions of the "inner state" alone of the substance, i.e. of the concentration $C=N / V$ and the temperature $T$. Since the same is of course true for the volume per unit particle $V / N=1 / C$, we see that $U$ may be regarded as a homogeneous function of the first degree in the three variables $S, V$ and $N$, so that, according to Euler's theorem

$$
U=T S-p V+\alpha N
$$

a particular case of a general relation which plays an important role in Gibbs' fundamental treatment of chemical equilibria. From (12) and (14) we get

$$
S d T-V d p+N d \alpha=0
$$

or, if by means of (14) we eliminate $S$,

$$
(U+p V-\alpha N)(d T / T)+N d \alpha=V d p,
$$

and thus

$$
d p=C[d \alpha-\alpha(d T / T)]+(u+p) d T / T,
$$

where as above $u=U / V$ denotes the energy density.

If after these preparations we return to our original problem and regard the quantities $\mu, p, C, T$ and $\alpha$ as functions of the coordinates we can take for the differentials in (15) the differences between corresponding quantities in two infinitely adjacent points of space and obtain thus from (15)

$$
\nabla p=C[\nabla \alpha-\alpha(\nabla T / T)]+(u+p) \nabla T / T,
$$

where $\nabla$ again denotes the gradient operator. Comparing this relation with the mechanical equilibrium condition (9) we get

$$
C[\nabla \alpha-\alpha(\nabla T / T)]+(u+p)\left[(\nabla T / T)+\frac{1}{2} \nabla \nu\right)=0 .
$$

We shall see that this equation contains two independent conditions for the equilibrium, one already found by Tolman, which corresponds to the equality of temperature everywhere in a classical equilibrium, the other corresponding to the Gibbs relation (1), which in the relativistic case are, however, curiously interrelated. In fact, if there are several, independent substances present in the same gravitational field an equation of the type (16) will hold for each of them separately with the same values of $\nu$ and $T$. As one such substance we shall always have the unordered temperature radiation, for which the chemical potential $\alpha$ vanishes. Thus we get

$$
\nabla T / T+\frac{1}{2} \nabla \nu=0 \quad \text { or } \quad T e^{\nu / 2}=\text { const., }
$$

which is the important relation governing the equilibrium in a gravitational field discovered by Tolman. ${ }^{4}$

\footnotetext{
${ }^{4}$ See reference 2, p. 318.
}

For substances with $\alpha \neq 0$ we get, moreover, from (16)

$\nabla \alpha / \alpha=\nabla T / T$ or $\alpha / T=$ const. or $\alpha e^{\nu / 2}=$ const.,

which together with (17) constitutes the relativistic analogon of the Gibbs relation (1).

Let us now regard the limiting case corresponding to ordinary Newtonian mechanics. Here we have with sufficient approximation $e^{\nu / 2}=1+\left(\varphi_{g} / c^{2}\right)$ and thus according to (13) and (18)

$$
\left(\mu+M c^{2}\right)\left[1+\left(\varphi_{g} / c^{2}\right)\right]=\text { const. }
$$

or in conformity with (1)

$$
\mu+M \varphi_{g}=\text { const., }
$$

where we have neglected the small term $\mu \varphi_{g} / c^{2}$. Still the application of (18) to (2) might at first sight seem puzzling, since we have also $\alpha / \theta=$ const. The relation $\theta\left[1+\left(\varphi_{g} / c^{2}\right)\right]=$ const. meaning, however, in. itself a negligible change of $\theta$ from point to point within the field, we may apply (1) in the same way as above, and obtain thus Eq. (3). On the other hand we may also regard the changes of $\theta$ as decisive. Thus according to (13) we have

$$
\frac{\mu}{\theta}=\frac{\alpha}{\theta}-\frac{M c^{2}}{\theta}, \frac{\mu_{0}}{\theta_{0}}=\frac{\alpha_{0}}{\theta_{0}}-\frac{M c^{2}}{\theta_{0}},
$$

with $\alpha / \theta=\alpha_{0} / \theta_{0}$ and $\theta\left[1+\left(\varphi_{\theta} / c^{2}\right)\right]=\theta_{0}$, which, in fact, gives

$$
\frac{\mu}{\theta}-\frac{\mu_{0}}{\theta_{0}}=M c^{2}\left(\frac{1}{\theta_{0}}-\frac{1}{\theta}\right)=-\frac{M \varphi_{g}}{\theta_{0}},
$$

in conformity with (3).

In order to apply relation (17) and (18) to a gravitational problem it is convenient to use the thermodynamic potential $\Omega$ given by

$$
\Omega=U-T S-\alpha N
$$

and regarded as a function of $T ; V$, and $\alpha$, which is most directly defined by means of Gibbs' grand ensemble. With this function given we obtain immediately $N, U$ and $p$ as functions of the same variables. In fact,

$$
N=-\frac{\partial \Omega}{\partial \alpha}, \quad U=\Omega-T \frac{\partial \Omega}{\partial T}-\alpha \frac{\partial \Omega}{\partial \alpha}, \quad p=-\frac{\partial \Omega}{\partial V},
$$

whereby $u=U / V$ and $p$ are functions of $\alpha$ and $T$ alone.

As an example we may regard a spherically symmetrical static field in the Einstein theory, where, using a polar coordinate system $r, \vartheta, \varphi$ and the time coordinate $t$, we may write for the line element

$$
d s^{2}=-e^{\lambda} d r^{2}-r^{2}\left(d \vartheta^{2}+\sin ^{2} \vartheta d \varphi^{2}\right)+e^{\nu} c^{2} d t^{2} .
$$

Apart from the relations (17) and (18) we have here 
the following two equations ${ }^{5}$ for $\lambda$ and $\nu$

$$
\begin{aligned}
& \kappa p=e^{-\lambda}\left(\frac{1}{r} \frac{d \nu}{d r}+\frac{1}{r^{2}}\right)-\frac{1}{r^{2}} \\
& \kappa u=e^{-\lambda}\left(\frac{1 d \lambda}{r} \frac{1}{d r}-\frac{1}{r^{2}}\right)+\frac{1}{r^{2}}
\end{aligned}
$$

$\kappa$ being the Einstein gravitational constant. Here $p$ and $u$ as functions of $\alpha$ and $T$ may according to (17) and (18) be expressed as functions of $\nu$. Further from the second Eq. (22) we get

$$
d / d r\left(r e^{-\lambda}\right)=1-\kappa r^{2} u,
$$

which together with the first Eq. (22) leads to the following differential equation in $\nu$ alone

$$
\frac{d}{d r}\left(\frac{1+\kappa r^{2} p}{d \nu / d r+1 / r}\right)=1-\kappa r^{2} u
$$

which with $p$ and $u$ given in terms of $\alpha$ and $\theta$ may be integrated numerically. As particular limiting cases we may consider that of a cold Fermi gas treated in detail by Oppenheimer and Volkoff ${ }^{6}$ and that of unordered

\footnotetext{
${ }^{5}$ See reference 2, p. 241.

${ }^{6}$ J. R. Oppenheimer and G. M. Volkoff, Phys. Rev. 55, 374 (1939).
}

temperature radiation treated by the present writer. ${ }^{7}$ In the former case $p$ and $u$ are functions of $\alpha$ alone. The expressions being somewhat complicated in the general case we shall only consider the case of zero rest mass. Here the pressure is one third of the energy density just as in the case of temperature radiation, which means that the gravitational problem is the same in the two cases. It is interesting to see how this follows by means of (18) and (17) respectively. Thus we have for a cold Fermi gas of zero rest mass

and thus

$$
\Omega=-\left(2 \pi / 3 h^{3} c^{3}\right) V \alpha^{4}
$$

$$
p=u / 3=\left(2 \pi / 3 h^{3} c^{3}\right) \alpha^{4}=p_{0} e^{-2 \nu},
$$

where $\nu$ is put equal to 0 at the point, where $p=p_{0}$, for instance at the centre.

In the second case they are functions of $\theta$ alone and we get

$$
p=u / 3=\left(8 \pi^{5} / 45 h^{3} c^{3}\right) \theta^{4}=p_{0} e^{-2 \nu} .
$$

We shall not pursue further the application of (23) and (24) in the two cases, which would hardly simplify the calculations made by the authors mentioned. The intention was only to point out how these problems might have been solved without the use of special artifices just by means of the general theorems (17) and (18).

${ }^{7}$ O. Klein, Arkiv f. mat., astr. o. fys. Bd. 34A, No. 19 (1947). 